
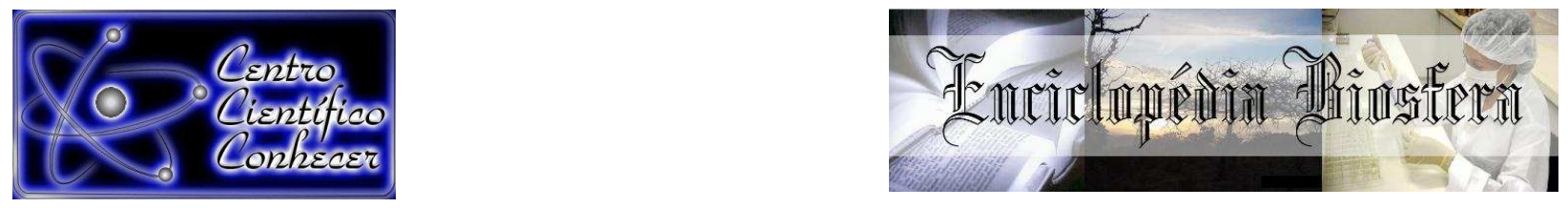

\title{
CRESCIMENTO E DESENVOLVIMENTO DE MUDAS DE Schizolobium amazonicum Huber ex Ducke EM DIFERENTES SUBSTRATOS E RECIPIENTES
}

Douglas Santos Gonçalves $^{1}$, Katiane Sousa de Carvalho², Patrícia Aparecida de Souza $^{3}$, Lúcia Vânia Ribeiro Lisboa ${ }^{2}$, André Ferreira dos Santos ${ }^{3}$

1 Mestrando em Ciências Florestais e Ambientais da Universidade Federal do Tocantins (goncalvesds@hotmail.com) Gurupi - Brasil

2 Graduandas em Engenharia Florestal da Universidade Federal do Tocantins 3 Professor (a) Dr. (a) do Curso de Engenharia Florestal da Universidade Federal do Tocantins

Recebido em: 08/04/2016 - Aprovado em: 30/05/2016 - Publicado em: 20/06/2016 DOI: 10.18677/Enciclopedia_Biosfera_2016_033

\begin{abstract}
RESUMO
Schizolobium amazonicum conhecida popularmente como paricá, é uma espécie amazônica de grande potencial econômico, e vem se destacando cada vez mais, tornando-se importante entre as espécies utilizadas em reflorestamentos no Brasil, pois possui desenvolvimentos com rapidez em altura e diâmetro, que possibilitam sua utilização em poucos anos e apresenta madeira com pequena quantidade de nós e defeitos, característica fundamental na sua utilização para fins estruturais. Objetivou-se neste trabalho avaliar a influência de diferentes substratos e recipientes no crescimento e desenvolvimento de Schizolobium amazonicum. O delineamento estatístico utilizado foi um DIC fatorial $2 \times 3$ (2 substratos e 3 recipientes), com 6 tratamentos e 10 repetições cada. Sendo T1(Terra de subsolo e recipiente pequeno), T2(Substrato comercial Bioflora ${ }^{\circledR}$ e recipiente pequeno) T3(Terra de subsolo e recipiente médio), T4(Substrato comercial Bioflora ${ }^{\circledR}$ e recipiente médio) e T5(Terra de subsolo e recipiente grande) e T6(Substrato comercial Bioflora ${ }^{\circledR}$ e recipiente grande). Avaliou-se aos 30,60, 90 e 120 dias após a semeadura, as variáveis: altura $(\mathrm{H})$, diâmetro do colo (D). Ao final do experimento, determinou-se a massa verde e matéria seca da raiz e parte aérea e o índice Qualidade de Dickson (IQD). Para a produção de mudas de Schizolobium amazonicum recomenda-se a utilização de substrato composto por terra de subsolo e sacos plásticos de $20 \times 30 \mathrm{~cm}$.
\end{abstract}

PALAVRAS-CHAVE: Paricá, Produção de mudas. Viveiro Florestal.

\section{GROWTH AND Schizolobium amazonicum Huber ex Ducke SEEDLINGS DEVELOPMENT IN DIFFERENT SUBSTRATES AND CONTAINERS}

\begin{abstract}
Schizolobium amazonicum popularly known as Paricá, is an Amazonian species of great economic potential, and has stood out increasingly important becoming among the reforested species in Brazil, as it has developments quickly in height and diameter, which allow its use in a few years and develops a small amount of wood with knots and flaws, a fundamental characteristic in its use for structural purposes. The aim of this study was to evaluate the influence of different substrates and ENCICLOPÉDIA BIOSFERA, Centro Científico Conhecer - Goiânia, v.13 n.23; p. 3782016
\end{abstract}


recipients in the growth and development of Schizolobium amazonicum. The statistical design was a factorial 2x3 DIC (2 substrates and 3 containers), with 6 treatments and 10 repetitions each. T1 (underground earth and $17 X 22 \mathrm{~cm}$ container), T2 (commercial substrate Bioflora ${ }^{\circledR}$ and $17 X 22 \mathrm{~cm}$ container) T3 (underground earth and $20 \times 30 \mathrm{~cm}$ container), T4 (commercial substrate Bioflora ${ }^{\circledR}$ and $20 \times 30 \mathrm{~cm}$ container) and T5 (subsurface earth and 25X30 cm container) and T6 (commercial substrate Bioflora ${ }^{\circledR}$ and $25 \times 30 \mathrm{~cm}$ container). It is assessed at 30,60, 90 and 120 days after sowing, the following variables: height $(\mathrm{H})$, stem diameter $(\mathrm{D})$. At the end of the experiment, it was determined the green mass and dry matter of root and shoot and Dickson Quality Index (IQD). For the production of Schizolobium amazonicum seedlings recommends the use of substrate composed of ground and underground plastic bags $20 \times 30 \mathrm{~cm}$.

KEYWORDS: Forest Nursery, Paricá, Seedling production

\section{INTRODUÇÃO}

A desproporção crescente entre oferta e demanda de madeira, nos mercados interno e externo, tem incentivado o setor industrial de base florestal para a utilização de madeiras oriundas de florestas plantadas. Assim, essas atividades têm aumentado nas áreas mais desenvolvidas do país, nas quais grandes expansões de terra são cultivadas, com espécies arbóreas de rápido crescimento (CORDEIRO et al., 2015).

O setor brasileiro de florestas plantadas no Brasil ocupa apenas 7,74 milhões de hectares, correspondendo a $0,9 \%$ do território brasileiro, sendo encarregado por $91 \%$ do total da madeira gerada para fins industriais no País - os demais 9\% procedem de florestas nativas manejadas adequadamente (IBÁ, 2015).

$A$ rede de produção do setor brasileiro de base florestal relacionado às florestas plantadas identifica-se pelo alto número de produtos, incluindo a produção, a colheita e o transporte de madeira, compreendendo também o alcance dos artigos finais nos segmentos industriais de Carvão vegetal e Biomassa, Siderurgia, Papel e Celulose, Painéis de Madeira Industrializada a Madeira Processada Mecanicamente, entre outros (ABRAF, 2013). Schizolobium amazanicum vem ganhado destaque entre as espécies cultivadas, apresentando características desejáveis para 0 mercado madeireiro.

A espécie é conhecida popularmente como paricá, ocorre na região amazônica, de grande potencial econômico, e vem se destacando cada vez mais, tornando-se importante entre as espécies reflorestadas no Brasil, pois possui desenvolvimentos com rapidez em altura e diâmetro, que possibilitam sua utilização em poucos anos e desenvolve uma madeira com pequena quantidade de nós e defeitos, característica fundamental na sua utilização para fins estruturais (ALMEIDA et al., 2013).

O paricá vem se destacando no cenário nacional como uma espécie promissora, com grande aceitação nos mercados externo e interno. Em 2014, a área plantada com a espécie correspondeu a cerca de 89.081 mil ha (IBÁ, 2015). Por esse motivo é de grande importância conhecer e facilitar os processos que envolvem a produção de mudas desta espécie.

Podendo ter influência positiva ou negativa na germinação das sementes e o crescimento das plântulas, o substrato utilizado é considerado muito importante para 
o desenvolvimento destas. É importante identificar os produtos encontrados nas diferentes regiões do país e torná-los disponíveis como substratos, reduzindo assim os custos de produção, o que eleva a magnitude em desenvolver substratos de fácil aquisição e utilização (GONDIN et al., 2015). Segundo PEREIRA et al., (2010) o recipiente escolhido traz efeitos de ordem técnica e econômica, sendo considerados os ideais aqueles que harmonizam o custo de produção.

Após a escolha dos substratos e recipientes faz-se necessário utilizar padrões para determinar a qualidade das mudas. Recomenda-se o uso das características morfológicas, que são baseadas tanto nos aspectos fenotípicos, quanto fisiológicas, que são definidas internamente na planta. Sendo mais utilizadas as características morfológicas, que determinam a qualidade das mudas e têm maior aceitação dos viveiristas (ELOY et al., 2013)

Partindo desse princípio objetivou-se neste trabalho avaliar a influência de diferentes substratos e recipientes no crescimento e desenvolvimento de Schizolobium amazonicum.

\section{MATERIAL E MÉTODOS}

O experimento foi conduzido de agosto a dezembro de 2015, no Viveiro da Universidade Federal do Tocantins, Campus de Gurupi - TO.

Clima tropical úmido com pequena deficiência hídrica (B1wA'a'), a temperatura média anual é de $29,5^{\circ} \mathrm{C}$, com precipita ção anual média de $1804 \mathrm{~mm}$, sendo um verão chuvoso e um inverno seco.

O lote das sementes de párica foi adquirido através do Grupo Caiçara, localizado em Brejo Alegre, no interior de São Paulo. A terra de subsolo foi coletada na área experimental da Universidade Federal do Tocantins - Câmpus Gurupi, a uma profundidade de $20-40 \mathrm{~cm}$ e peneirada em malha de $3 \mathrm{~mm}$. A terra de subsolo é utilizada para formação de mudas, por estar isenta de sementes de plantas indesejáveis e de microrganismos patogênicos, são provenientes de barrancos. Porém podem apresentar baixa fertilidade ou desequilíbrio nutricional (SILVA \& MORAIS, 2013). O substrato comercial Bioflora ${ }^{\circledR}$ foi comprado em casas agropecuárias da cidade. A análise química dos substratos utilizados no experimento pode ser observada na tabela 1.

TABELA 1 - Resultado da análise química dos substratos utilizados no experimento.

\begin{tabular}{lcccrcc}
\hline Amostra & $\mathrm{N}$ & $\mathrm{P}$ & $\mathrm{K}$ & $\begin{array}{c}\mathrm{Ca} \\
\mathbf{g} / \mathbf{k g}\end{array}$ & $\mathrm{Mg}$ & $\mathrm{S}$ \\
& & \multicolumn{7}{c}{0,0} & 0,4 & 0,1 & 0,1 \\
\hline Terra de Subsolo & 1,4 & 0,9 & 1,0 & 9,1 & 2,0 & 1,2 \\
Comercial Bioflora $^{\circledR}$ & 4,7 & 1,4 & 0,8 & 9,8 \\
\hline
\end{tabular}

A análise química dos substratos (Tabela 1) foi realizada de acordo com a metodologia de (TEDESCO, 1995), no Laboratório de Solos a Universidade Federal do Tocantins e laboratório especializado localizados em Gurupi, TO.

As sementes foram selecionadas e armazenadas em câmaras frias. Em seguida, foi realizada a superação de dormência das mesmas, por escarificação mecânica, com o uso de esmeril mecânico. A escarificação foi feita próximo à região do eixo embrionário, colocando-o de encontro à lixa do esmeril, fazendo uma ruptura 
parcial do tegumento, realizando um dia antes da semeadura, no dia 24 de agosto de 2015.

Os tratamentos consistiram da combinação de dois tipos de substratos e três tamanhos de recipientes, totalizando seis tratamentos, cada um foi composto por dez repetições, tabela 2 .

TABELA 2 - Descrição dos tratamentos testados no experimento.

\begin{tabular}{lll}
\hline Tratamentos & Recipientes & Substratos \\
\hline T1 & $17 \times 22 \mathrm{~cm}$ & Terra de Subsolo \\
T2 & $17 \times 22 \mathrm{~cm}$ & Comercial Bioflora $^{\circledR}$ \\
T3 & $20 \times 30 \mathrm{~cm}$ & Terra de Subsolo \\
T4 & $20 \times 30 \mathrm{~cm}$ & Comercial Bioflora $^{\circledR}$ \\
T5 & $25 \times 30 \mathrm{~cm}$ & Terra de Subsolo \\
T6 & $25 \times 30 \mathrm{~cm}$ & Comercial Bioflora $^{\circledR}$ \\
\hline
\end{tabular}

Os sacos plásticos foram preenchidos manualmente e receberam duas sementes, colocadas a uma profundidade de $3 \mathrm{~cm}$. As mudas foram acondicionadas em viveiro florestal, com tela sombrite de $50 \%$, sendo a irrigação manual realizada duas vezes ao dia. Logo após a germinação, foi realizado o raleio, deixando sempre que possível a plântula com maior vigor e a mais centralizada no recipiente.

Aos 30, 60, 90 e 120 dias, após a semeadura foram avaliados parâmetros: a) altura da planta $(\mathrm{H})$ com régua graduada em centímetros $(\mathrm{cm})$; b) diâmetro do colo (DC) a cm do substrato, com paquímetro de plástico graduado em milímetros $(\mathrm{mm})$.

Para a determinação de massa seca da parte aérea (MSPA) e massa seca da raiz (MSR), as plantas foram retiradas dos recipientes, lavadas cuidadosamente e fracionadas em parte aérea e raiz. O material vegetal foi seco a $75^{\circ} \mathrm{C}$ em estufa de circulação forçada até atingir peso constante, realizando posteriormente à pesagem do material em balança analítica eletrônica.

Para a determinação da qualidade das mudas utilizou-se o Índice de qualidade de Dickson (IQD) (DICKSON et al., 1960), através da fórmula a seguir:

$$
I Q D=\frac{\text { MST }(\mathrm{g})}{\frac{\mathrm{H}(\mathrm{cm})}{\mathrm{DC}(\mathrm{mm})}+\frac{\text { PMSPA }(\mathrm{g})}{\operatorname{PMSR}(\mathrm{g})}}
$$

Sendo: IQD: Índice de qualidade de Dickson; MST: Massa seca total (g); H: Altura $(\mathrm{cm})$; DC: Diâmetro do colo $(\mathrm{mm})$; PMSPA: Peso da matéria seca da parte aérea $(\mathrm{g})$; PMSRA: Peso da matéria seca da raiz (g).

Os efeitos causados sobre as variáveis estudadas nas plantas de Schizolobium amazonicum foram submetidos a análise de variância e as medias foram comparadas pelo teste de Tukey ao nível de $5 \%$ de probabilidade, utilizando o software Assitat 7.7 beta (SILVA, 2015). 


\section{RESULTADOS E DISCUSSÃO}

Aos 30 e 60 dias após a semeadura os tratamentos T2(Sacos de $17 X 22 \mathrm{~cm}$ e subst. comercial) e T4(Sacos de $20 \times 30 \mathrm{~cm}$ e subst. comercial) não diferiram significativamente entre si apresentando as maiores médias para altura $(\mathrm{H})$. Aos 90 e 120 dias para a variável altura não houve diferença significativa entre os tratamentos testados, mostrando que para esta variável, todos os tratamentos foram adequados (tabela 3 ). Isso mostra que para o desenvolvimento em altura a quantidade dos nutrientes citados na tabela 1, em ambos os tratamentos foram satisfatórios.

A altura das plantas $(\mathrm{H})$ é tecnicamente aceita como boa medida do provável comportamento das mudas e o padrão de qualidade possui elevada correlação com o diâmetro de colo, originando maiores taxas de sobrevivência e de crescimento em campo (PUÉRTOLAS et al., 2012). Proporcionando uma ótima estimativa da predição do crescimento inicial no campo.

TABELA 3 - Média dos tratamentos para altura $(\mathrm{H}) \mathrm{cm}$ e diâmetro $(\mathrm{D}) \mathrm{mm}$ de mudas de Schizolobium amazonicum aos 30, 60,90 e 120 dias após a semeadura.

\begin{tabular}{|c|c|c|c|c|c|c|c|c|}
\hline Tratamentos & $\begin{array}{l}\mathrm{H}(\mathbf{3 0} \\
\text { Dias) }\end{array}$ & $\begin{array}{l}\text { D (30 } \\
\text { Dias) }\end{array}$ & $\begin{array}{l}\mathbf{H}(60 \\
\text { Dias) }\end{array}$ & $\begin{array}{l}\text { D (60 } \\
\text { Dias) }\end{array}$ & $\begin{array}{l}\mathrm{H} \text { (90 } \\
\text { Dias) }\end{array}$ & $\begin{array}{l}\text { D (90 } \\
\text { Dias) }\end{array}$ & $\begin{array}{l}\mathrm{H}(\mathbf{1 2 0} \\
\text { Dias) }\end{array}$ & $\begin{array}{l}\mathrm{D}(120 \\
\text { Dias) }\end{array}$ \\
\hline T1 & $14,92 \mathrm{~b}$ & $3,1 \mathrm{a}$ & $15,88 \mathrm{~b}$ & $3,8 \mathrm{a}$ & $19,17 \mathrm{a}$ & $4,4 a$ & $21,34 \mathrm{a}$ & $5,2 \mathrm{a}$ \\
\hline T2 & $19,69 \mathrm{a}$ & $2,8 \mathrm{a}$ & $21,77 \mathrm{a}$ & $3,4 \mathrm{a}$ & $25,09 \mathrm{a}$ & $4,2 \mathrm{a}$ & 25,99 a & $4,2 \mathrm{~b}$ \\
\hline T3 & $14,58 \mathrm{~b}$ & 3,3 a & $18,44 \mathrm{~b}$ & $4,2 \mathrm{a}$ & $23,82 \mathrm{a}$ & $5,5 \mathrm{a}$ & $25,40 \mathrm{a}$ & $6,0 \mathrm{a}$ \\
\hline T4 & $22,71 \mathrm{a}$ & $3,3 \mathrm{a}$ & $25,65 \mathrm{a}$ & $4,0 \mathrm{a}$ & $29,10 \mathrm{a}$ & $4,1 \mathrm{a}$ & $29,28 \mathrm{a}$ & $4,5 \mathrm{a}$ \\
\hline T5 & $15,77 b$ & $2,9 a$ & $19,15 b$ & $4,2 \mathrm{a}$ & $22,74 \mathrm{a}$ & $5,0 \mathrm{a}$ & $25,45 \mathrm{a}$ & $5,7 \mathrm{a}$ \\
\hline T6 & $17,57 \mathrm{~b}$ & $2,9 a$ & $19,04 \mathrm{~b}$ & $3,4 a$ & $19,46 \mathrm{a}$ & $3,7 \mathrm{a}$ & $20,57 \mathrm{a}$ & $4,0 \mathrm{~b}$ \\
\hline CV(\%) & 26,53 & 23,82 & 24,26 & 23,62 & 29,33 & 27,88 & 28,56 & 25,97 \\
\hline
\end{tabular}

As médias seguidas pela mesma letra não diferem estatisticamente entre si pelo teste de Tukey ao nível de $5 \%$ de probabilidade

Para o diâmetro observou-se diferença significativa apenas aos 120 dias, onde os tratamentos T1(Sacos de 17X22 cm e subst. subsolo), T3(Sacos de $17 X 22 \mathrm{~cm}$ e subst. subsolo), T4(Sacos de $20 \times 30 \mathrm{~cm}$ e subst. comercial) e T5(Sacos de $25 \times 30$ $\mathrm{cm}$ e subst. subsolo) exibiram as maiores médias (tabela 3).

Segundo CARVALHO (2007) as mudas de Shcizolobium amazonicum atingiram tamanho adequado para plantio em campo entre $20 \mathrm{~cm}$ a $35 \mathrm{~cm}$ de altura, cerca de 60 dias após a semeadura. No presente estudo os tratamentos T2(Sacos de $17 X 22 \mathrm{~cm}$ e subst. comercial) e T4(Sacos de $20 X 30 \mathrm{~cm}$ e subst. comercial) atingiram média de 21,77 e 25,65 cm, respectivamente, mostrando que aos 60 dias após a semeadura o substrato ideal foi o comercial Bioflora ${ }^{\circledR}$ (tabela3), pois este apresenta maiores quantidades de nutrientes (Tabela 1), o que pode ter influenciando positivamente no desenvolvimento das mudas nos dois primeiros meses, quando comparado com o substrato terra de subsolo, de baixa fertilidade (Tabela 1).

Schizolobium amazonicum pertence ao grupo ecológico das espécies pioneiras (SWAINE; WHITMORE, 1988). Podendo ocorrer em solos de baixa fertilidade, como 
pôde ser observado no T1(Sacos de 17X22 cm e subst. Subsolo) no qual o substrato utilizado foi terra de subsolo, que geralmente apresenta baixa fertilidade (Tabela 1), onde não foi realizado nenhum tipo de adubação. Neste tratamento a espécie apresentou altura de $21,34 \mathrm{~cm}$ e $5,2 \mathrm{~mm}$ (tabela 3). A espécie se desenvolve em temperaturas médias de $27^{\circ} \mathrm{C}$ e uma precipitação entre $1.600 \mathrm{~mm}$ a $3.000 \mathrm{~mm}$ (CARVALHO, 2007). O que pode explicar o bom desenvolvimento da espécie na maioria dos tratamentos testados, pois de acordo com Klink e Machado (2005) as temperaturas na região geralmente ao longo do ano variam, entre $22^{\circ} \mathrm{C}$ e $28^{\circ} \mathrm{C}$ em média e a precipitação média anual é de $1.500 \mathrm{~mm}$ a $1.600 \mathrm{~mm}$.

MATOS et al., (2009) analisaram o desenvolvimento das mudas de Schizolobium amazonicum em sacos de polietileno $(12 \mathrm{~cm} \times 5 \mathrm{~cm})$, com substrato não comercial, submetidas a $75 \%$ de sombreamento, e constataram que essas tiveram maior desenvolvimento, pois, aos 90 dias após a semeadura, apresentaram $28,8 \mathrm{~cm}$ de altura e $3,9 \mathrm{~mm}$ de diâmetro do colo. Os mesmos autores relatam que as condições climáticas do local apresentavam temperatura média menor do que a região de origem da espécie pode ter influenciando no desenvolvimento das mudas em altura e diâmetro do colo. Evidenciado que o clima da região de estudo afetou positivamente o desenvolvimento das mudas, pois aos 90 dias, médias de altura de $29,28 \mathrm{~cm}$ e diâmetro de $4,1 \mathrm{~mm}$, foram maiores, se comparadas a dos referidos autores.

FRIGOTO et al., (2015) avaliando o desenvolvimento de Shcizolobium amazonicum em diferentes ambientes no viveiro, com mudas produzidas em saco plástico de polietileno $(12 \times 5 \mathrm{~cm})$, contendo substrato compostos por: terra de subsolo (50\%), areia lavada (25\%) e cama de aviário (25\%), verificaram que as mudas da espécie em casa de sombra $(75 \%)$ e em casa de vegetação apresentaram as maiores medias de altura e diâmetro aos 280 dias. Relatando que a condução do estudo em viveiro sombreado (50\%) pode ter favorecido o desenvolvimento da espécie.

LANGE et al., (2014) avaliando substratos orgânicos para produção de mudas de Schizolobium amazonicum, relatam que aos 90 dias após a semeadura as mudas apresentaram as maiores medias de altura $(38,75 \mathrm{~cm}$ e $41,27 \mathrm{~cm})$ e diâmetro $(4,02$ $\mathrm{mm}$ e $4,09 \mathrm{~mm}$ ) nos tratamentos que utilizaram substrato contendo $20 \%$ de moinha de carvão; $40 \%$ de serragem; $20 \%$ de esterco bovino e $20 \%$ de esterco de aves; e o substrato contendo $20 \%$ de moinha de carvão; $20 \%$ de serragem; $40 \%$ de esterco bovino e $20 \%$ de esterco de aves, respectivamente.

No presente no estudo, as mudas de paricá 90 dias após a semeadura apresentaram altura média de $29,10 \mathrm{~cm}$ e diâmetro de $4,10 \mathrm{~mm}$, demostrando a importância da adição de substratos orgânicos na produção de mudas dessa espécie, quando se compara com os resultados obtidos por LANGE et al., (2014).

ANTONIAZZI et al., (2013), avaliando a eficiência de recipientes no desenvolvimento de mudas de Cedrela fissilis, observou que aos 120 dias que as mudas cultivadas em cinco diferentes recipientes tiveram diminuição nos parâmetros de crescimento com a diminuição do tamanho do recipiente. $E$ que as maiores médias de altura e diâmetro foram encontradas no tratamento que utilizou como recipiente o saco plástico grande $\left(1.962,5 \mathrm{~cm}^{3}\right)$. Assim, nossos resultados diferiram destes, pois aos 120 dias houve diferença significativa entre os tratamentos apenas na variável diâmetro, ou seja, o tamanho do recipiente não influi no desenvolvimento das mudas. 
A massa seca segundo GOMES e PAIVA (2006) pode ser entendida como a rusticidade de uma muda, ou seja, quanto maior for a muda mais rústica estará, e em condições favoráveis de irem ao campo, as mudas devem estar livres de microrganismos e aptas, para assim, promover uma máxima sobrevivência, minimizando gastos.

O tratamento T3(Sacos de $20 \times 30 \mathrm{~cm}$ e subst. subsolo) apresentou a maior média em relação à matéria seca da parte aérea (MSPA) com 4,96 g, diferindo significativamente dos demais. Para a matéria seca da raiz não houve diferença significativa entre os tratamentos (Tabela 4).

TABELA 4 - Médias de peso da massa verde da parte aérea (MSPA), massa verde da raiz (MVR), massa seca da parte aérea (MSPA), massa seca da raiz (MSR), e Índice Qualidade de Dickson (IQD) de Schizolobium amazonicum aos 120 dias após a semeadura.

\begin{tabular}{cccccc}
\hline TRATAMENTOS & \multicolumn{7}{l}{} \\
\hline \multicolumn{7}{c}{ MVPA(g) } & MVR(g) & MSPA(g) & MSR(g) & IQD \\
\hline T1 & $4,33 \mathrm{~b}$ & $1,94 \mathrm{a}$ & $1,73 \mathrm{abc}$ & $1,34 \mathrm{a}$ & $0,07 \mathrm{abc}$ \\
T2 & $3,96 \mathrm{~b}$ & $3,75 \mathrm{a}$ & $1,50 \mathrm{bc}$ & $1,46 \mathrm{a}$ & $0,04 \mathrm{bc}$ \\
T3 & $10,83 \mathrm{a}$ & $3,75 \mathrm{a}$ & $4,96 \mathrm{a}$ & $2,00 \mathrm{a}$ & $0,15 \mathrm{a}$ \\
T4 & $4,09 \mathrm{~b}$ & $1,87 \mathrm{a}$ & $1,42 \mathrm{bc}$ & $1,20 \mathrm{a}$ & $0,04 \mathrm{bc}$ \\
T5 & $9,92 \mathrm{a}$ & $2,90 \mathrm{a}$ & $4,54 \mathrm{bc}$ & $1,64 \mathrm{a}$ & $0,13 \mathrm{ab}$ \\
T6 & $2,49 \mathrm{~b}$ & $1,49 \mathrm{a}$ & $0,98 \mathrm{c}$ & $0,71 \mathrm{a}$ & $0,03 \mathrm{c}$ \\
CV (\%) & 78,74 & 74,04 & 72,55 & 77,01 & 74,22 \\
\hline
\end{tabular}

As médias seguidas pela mesma letra não diferem estatisticamente entre si pelo teste de Tukey ao nível de 5\% de probabilidade.

LANGE et al., (2014) avaliando quatro substratos orgânicos para produção de mudas de Schizolobium amazonicum, verificaram que aos 90 dias após a semeadura, que o substrato contendo $20 \%$ de moinha de carvão; $26,66 \%$ de serragem; 26,66\% de esterco bovino; 26,66\% de esterco de aves, proporcionou maior acúmulo de matéria seca de raízes $(9,26 \mathrm{~g})$ e para matéria seca da parte aérea não houve diferença significativa entre os tratamentos $(5,15 \mathrm{~g} ; 5,09 \mathrm{~g}, 5,38 \mathrm{~g} \mathrm{e}$ $4,84 \mathrm{~g}$ ). Os resultados apresentados neste estudo foram valores inferiores (tabela 4) aos encontrados pelos autores para os mesmos parâmetros avaliados, confirmando a importância da adição de compostos orgânicos para aumentar o incremento de biomassa para espécie.

Uma vez que o peso da parte aérea tem correlação direta com a sobrevivência e desempenho inicial das mudas após o plantio, sendo um indicador de rusticidade de plantas. A matéria seca da raiz está relacionada a um maior desenvolvimento radicular o que se torna benéfico para o crescimento das mudas em campo (GOMES \& PAIVA 2006).

ALVES, et al., (2012) avaliando diferentes tamanhos de recipientes e tipos de substratos na produção de mudas de Anadenanthera macrocarpa, notaram que não existiu diferença significativa dos recipientes e substratos testados, tanto para a massa seca da parte aérea e quanto para massa seca da raiz. 
Para o índice de qualidade de Dickson o T3(Terra de subsolo e recipiente médio) apresentou a maior média, diferindo significativamente dos demais tratamentos. AZEVEDO et al., (2010) relataram que o IQD é um ótimo indicador da qualidade de mudas, porque leva em conta em seu cálculo a robustez e o equilíbrio da distribuição da biomassa na muda, ponderando os resultados de parâmetros importantes utilizados na avaliação da qualidade de mudas, como a altura, o diâmetro, matéria seca parte aérea, raiz e também a total.

Em relação às estimativas do IQD, verificou-se que os valores obtidos foram inferiores aos citados por CHAVES \& PAIVA (2004), de 0,20. Evidenciando assim que para o IQD, o tratamento que mais se aproximou do valor adequado foi 0 T3(Sacos de 20X30 cm e subst. subsolo), 0,15.

O valor do IQD para mudas de Schizolobium amazonicum, foi de 3,14 quando LANGE et al., (2014) utilizaram substratos orgânicos contendo $20 \%$ de moinha de carvão; 26,66\% de serragem; 26,66\% de esterco bovino; $26,66 \%$ de esterco de aves, avaliados aos 90 dias após a semeadura.

\section{CONCLUSÃO}

Para a produção de mudas de Schizolobium amazonicum recomenda-se a utilização de substrato composto por terra de subsolo e sacos plásticos de $20 \times 30 \mathrm{~cm}$.

\section{REFERÊNCIAS}

ASSOCIAÇÃO BRASILEIRA, DE PRODUTORES DE FLORESTAS PLANTADAS ABRAF. Anuário estatístico da ABRAF 2013, Ano base 2012. Brasília, 2013. 142 p.

AZEVEDO, I. M. G.; AlenCAR, R. M.; BARBOSA, A. P.; ALMEIDA, N. O. Estudo do crescimento e qualidade de mudas de marupá (Simarouba amara Aubl.) em viveiro. Acta Amazônica, Manaus, v. 40, n.1, p. 157-164, 2010. Disponível em: <http://dx.doi.org/10.1590/S0044-59672010000100020>. doi: 10.1590/S004459672010000100020

ALMEIDA, H. D.; Scaliantell, R. de. M.; Macedo, L. B. de.; Macêdo, A. N.; Dias, A. A.; Christoforo, A. L.; Junior, C. C. Caracterização completa da madeira da espécie Amazônica Paricá (Schizolobium amazonicum Herb) em peças de dimensões estruturais. Revista Árvore, Viçosa, v.37, n.6, p.1175-1181, 2013. Disponível em: <http://dx.doi.org/10.1590/S0100-67622013000600019>. doi: 10.1590/S010067622013000600019.

ALVES, A.S.; OLIVEIRA, L.S.B.; ANDRADE, L.A.; GONÇALVES, G.S.; SILVA, S.M. Produção de mudas de angico em diferentes tamanhos de recipientes e composições de substratos. Revista Verde, Mossoró, v.7, n.2, p.39-44, 2012. Disponível em: <http://www.gvaa.com.br/revista/index.php/RVADS/article/view/ $1108 / 1153>$.

ANTONIAZZI, A. P., BINOTTO, B., NEUMANN, G. M., S, T. L., B, J. C. Eficiência de recipientes no desenvolvimento de mudas de Cedrela fissilis Vell. (Meliaceae). Revista Brasileira de Biociências, Porto Alegre, v. 11, n. 3, p.313317, set. 2013. Disponível em: <www.ufrgs.br/seerbio/ojs/index.php/rbb/ article/view/2390>. 
CARVALHO, P.E.R. Paricá - Schizolobium amazonicum. Colombo: Embrapa Florestas, 2007. 8p. (Embrapa-CNPF. Circular Técnica, 142).

CHAVES, A. S.; PAIVA, H. N. Influência de diferentes períodos de sombreamento sobre a qualidade de mudas de fedegoso (Senna macranthera (Collad.) Irwin et Barn). Scientia Forestalis, Piracicaba, n. 65, p. 22 - 29, 2004.

CORDEIRO, I. M. C. C., BARROS, P. L. C. de., LAMEIRA, O. A., GAZEL FILHO, A. B. Avaliação de plantios de paricá (Schizolobium parahyba var. amazonicum (Huber ex Ducke) Barneby de diferentes idades e sistemas de cultivo no município de Aurora do Pará - PA (Brasil). Ciência Florestal, Santa Maria, v. 25 n. 3, p. 679 687. Jul/Set. 2015. Disponivel em: <http://dx.doi.org/10.5902/1980509819618>. doi: 10.5902/1980509819618.

DICKSON, A.; LEAF, A.L.; HOSNER, J.F. Quality appraisal of white spruce and white pine seedling stock in nurseries. Forest. Chronicles, v. 36, p. 10-13, 1960.

ELOY, E., CARON, B. O., SCHMIDT, D., BEHLING, A., SCHWERS, L., ELLI, E. F. Avaliação da Qualidade de Mudas de Eucalyptus Grandis Utilizando Parâmetros Morfológicos. Floresta, Curitiba, PR, v. 43, n. 3, p. 373 - 384, jul. / set. 2013. Disponível em: <http:dx.doi.org/10.5380/rf.v43i3.26809>. doi: 10.5380/rf.v43i3.26809.

FRIGOTTO, T., BRUN, E. J., MEZZALIRA, C. C., NAVROSKI, M. C., BIZ, S., RIBEIRO, R. R. Desenvolvimento de mudas de Schizolobium amazonicum Huber ex Ducke em diferentes ambientes em viveiro. Ecologia e Nutrição Florestal, Santa Maria-RS, v.3, n.1, p.09-17, jan./abr., 2015. Disponível em: < http://dx.doi.org/10.5902/2316980X17061 >. doi: 10.5902/2316980X17061.

GOMES, J.M.; PAIVA, H.N. Viveiros Florestais: propagação sexuada. 1. ed. Viçosa: UFV, 2006. $116 \mathrm{p}$.

GONDIN, J. C., SILVA, J. B. DA., ALVES, C. Z., DUTRA, A. S., JUNIOR, L. E. Emergência de plântulas de Schizolobium amazonicum Huber ex Ducke (Caesalpinaceae) em diferentes substratos e sombreamento. Revista Ciência Agronômica, Fortaleza, v.46, n.2, p 329 - 338, Abr./Jun. 2015. Disponível em: $<$ http://www.ccarevista.ufc.br/seer/index.php/ccarevista/article/view/3718/1179>.doi: 10.5935/1806-6690.20150012.

INDÚSTRIA BRASILEIRA DE ÁRVORES - IBÁ. Anuário estatístico 2015: ano base 2014. Brasília, 2015. 80 p. Disponível em: <http://iba.org/images/shared/iba_2015.pdf>. Acesso em: 29 jan. 2016.

KLINK C. A., MACHADO R. B. A conservação do Cerrado brasileiro. Brasília: Megadiversidade, 2005. 320p. 
LANGE, A., SILVA JUNIOR, J. G. DA.; CAIONE, G. Substratos para produção de mudas de Schizolobium amazonicum. Revista Tecnologia \& Ciência Agropecuária, João Pessoa, v. 8, n. 1, p.49-54, mar. 2014. Disponível em: < http://dx.doi.org/10.5935/1806-6690.20150012>. doi: 10.5935/1806-6690.20150012

MATOS, G. D. de., FRIGOTTO, T., MARTINS, A. P. M., BRUN, E. J. Desenvolvimento de mudas de paricá (Schizolobium amazonicum Huber ex Ducke) em substrato orgânico - estudo de caso. Synergismus scyentifica, Pato Branco, v.4, n.1, p.1 - 3, 2009.

PEREIRA, P. C., MELO, B. de., FREITAS, R. S. de., TOMAZ, M. A. Tamanho de recipientes e tipos de substrato na qualidade de mudas de tamarindeiro. Revista Verde, Mossoró, v. 5, n. 3, p. 136-142, 2010.

PUÉRTOLAS, J., JACOBS, D. F., BENITO, L. F., PEÑUELAS, J. L. 2012. Costbenefit analysis of different container capacities and fertilization regimes in Pinus stock-type production for forest restoration in dry Mediterranean areas. Ecological Engineering, v. 44, p 210-215. Disponível em: < http://www.sciencedirect.com/science/article/pii/S0925857412001231 >. doi: 10.1016/j.ecoleng.2012.04.005

SILVA, A. L.; MORAIS, G. A. Influência de diferentes substratos no crescimento inicial de Ormosia arbórea (Vell.) Harms (Fabaceae). Revista Verde de Agroecologia e Desenvolvimento Sustentável, Mossoró - RN, v. 8, n. 4, p. 22-27, out - dez, 2013. Disponível em: < http://www.gvaa.com.br/revista/index.php/RVADS/article/view/2243/1825>

SILVA, F. A. S. ASSISTAT: Versão 7.7 beta. DEAG-CTRN-UFCG - Atualizado em 01 de abril de 2015.

SWAINE, M. D.; WHITMORE, T. C. On the definition of ecological species groups in tropical rain forest. Vegetation, v.75, n, 1, p.81-86, 1988. Disponível em: < http://link.springer.com/article/10.1007\%2FBF00044629>. doi: 10.1007/BF00044629

TEDESCO, M. J. et al. Análise de solo, plantas e outros materiais. 2.ed. Porto Alegre, Departamento de Solos da Universidade Federal do Rio Grande do Sul. 1995. 174p. (Boletim Técnico de Solos, 5). 